\title{
RELIABILITY TEST DEMONSTRATION METHOD FOR EXPONEN- TIAL LIFE SYSTEM WITH RELIABILITY GROWTH UNDER THE CONDITION OF IN-TIME CORRECTIVE STRATEGY
}

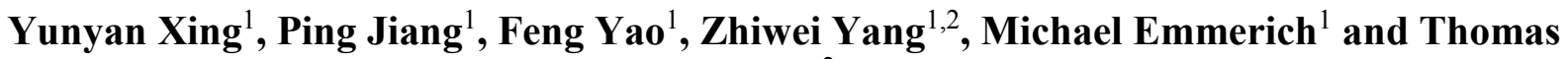 \\ Bäck $^{2}$ \\ ${ }^{1}$ National Univeristy of Defence Techonology \\ Deya road, No.109, Kaifu district, Changsha, Hunan, China \\ e-mail: xingyy2005@sina.com,jiangping@nudt.edu.cn,yf_nudt@126.com \\ ${ }^{2}$ Leiden Institute of Advanced Computer Science, \\ Niels Bohrweg 1, Leiden, 2333CA, the Netherlands \\ \{z.yang,m.t.m.emmerich,t.h.w.baeck\}@liacs.leidenuniv.nl
}

Keywords: Bayesian methods, reliability growth, exponential life system, In-Time corrective strategy, hypothesis testing.

\begin{abstract}
The development process of weapon system usually composes of multiple test stages. Especially for expensive weapon system which test is destructive, engineers often apply In-Time corrective strategy to analyze and modify failures exposed during development. That is to say once failure is observed at each stage, test is then stopped and a design modification is introduced to remove the cause of the observed failure, and thus release improved system to new test stage until the system reliability level satisfy the predefined development requirement. In this paper, we present a Bayesian statistical decision method to judge whether system reliability level meet development requirement or not, based on multiple stages test data during development of exponential life system and taking In-Time corrective strategy into account. Firstly, the reliability growth model, derived from non-homogeneous Poisson process, under the condition of In-Time corrective strategy is presented. Then prior distribution of system reliability index is introduced by applying reliability growth model to incorporate various test data from development process, and the Bayesian hypothesis testing method is given. Finally, the numerical example validates effectivity of the method compared with traditional methods.
\end{abstract}




\section{INTRODUCTION}

For most complex repairable system, the first prototype usually contains design and/or engineering flaws, thus the system has to experience an iterative process of TAAF to eliminate the observed failure modes and then achieve the reliability growth. Reliability growth model is an effective means to analyze the growth trend of product reliability, and many scholars has made lots of research. Fries [1], [2] presented a discrete reliability growth model, derived from the learning-curve property, to describe reliability growth. Xing [3] presented a discrete reliability growth model base on In-Time correction strategy. Hall \& Mosleh [4] introduced a framework for the evaluation of reliability growth for one-shot systems. Pulcini [5], [6], [7] proposed an exponential reliability growth model where several identical copies of equipment are put on test, once a failure is observed, design modification for all copies of equipment is introduced. In addition, Bayesian reliability growth models are very popular in describing the reliability growth trend of product [8], [9], [10], [11]. However, those reliability growth models are usually used to evaluate or predict product reliability index, little effort focused on hypothesis testing for whether the product reliability index has reached the predefined requirement during development.

As for weapon equipment, development process generally consist of multiple test stages. Especially for expensive system which test is destructive, test is stopped and design modifications are made immediately once failure is observed at each stage, such a corrective strategy for failure mode is often referred to as In-Time corrective strategy. While under the condition of In-Time corrective strategy, how to describe the reliability growth trend of system and how to judge whether the system has achieved the predefined reliability goal are prominent problems that reliability researchers concern about. So we make following four assumptions:

(i) System development process composes of multiple test stages, test data from various stages are independent.

(ii) In-Time corrective strategy is adopted to fix observed system failure at each test stage.

(iii) Test data at the ith $(i=1,2, \cdots, m)$ stage is $\left(1, \tau_{i}\right)$. Where 1 is the number of failure at the ith stage. $\tau_{i}$ denotes the time on test, and it is r. v. .

(iv) System reliability level at the ith stage is changeless, and system life $t$ conform to exponential distribution with parameter $\lambda_{i}$, i. e.

$$
f\left(t \mid \lambda_{i}\right)=\lambda_{i} e^{-\lambda_{i} t}, \quad \lambda_{i}, t>0, \quad i=1,2, \cdots
$$

Where $\lambda_{i}$ is the system failure rate at the ith stage.

The paper is organized as follows. A reliability growth model under the condition of InTime corrective strategy is presented In Section 1. The Bayesian hypothesis testing method is introduced in Section 2. A numerical example is given in Section3. Section 4 summarizes the paper.

\section{RELIABILITY GROWTH MODEL UNDER THE CONDITION OF IN-TIME CORRECTIVE STRATEGY}

Crow proposed AMSAA model which thought the cumulative number of observed failures $K(t)$ of a repairable system in the development duration $(0, t]$ follows the non-homogeneous Poisson process (NHPP) with the expectation of $K(t)$ as $E[K(t)]=c t^{d}$ and the probability is 


$$
P\{K(t)=n\}=\frac{\left(c t^{d}\right)^{n}}{n !} e^{-c t^{d}}, \quad n=0,1,2, \cdots
$$

Where parameters $c, d$ denote shape parameter and scale parameter respectively. When $0<d<1$, system reliability is increased. $1-d$ denotes growth rate.

In practice, people are more concerned with analysis on time on test $T(n)$ when $n$ failures happen, especially for analysis on the expectation of time on test $E[T(n)]$. It is obvious that $n$ failures happen before moment $t$ equals if and only if the number of failures until $t$ is more than $n$, namely,

$$
K(t) \geq n \Leftrightarrow T(n) \leq t
$$

So the distribution function $F_{T(n)}(t)$ of $T(n)$ is

$$
F_{T(n)}(t)=P\{T(n) \leq t\}=P\{K(t) \geq n\}=\sum_{j=n}^{\infty} e^{-c t^{d}} \frac{\left(c t^{d}\right)^{j}}{j !}
$$

Thus the probability density function $f_{T(n)}(t)$ of $T(n)$ is obtained by computing the derivative of $t$ from (4) as

$$
f_{T(n)}(t)=e^{-c t^{d}} \frac{\left(c t^{d}\right)^{n-1}}{(n-1) !} c d t^{d-1}
$$

So the expectation of time on test $E[T(n)]$ when $n$ failures happen is

$$
E[T(n)]=\int_{0}^{\infty} t f_{T(n)}(t) d t=\frac{d}{(n-1) !} \int_{0}^{\infty}\left(c t^{d}\right)^{n} e^{-c t^{d}} d t
$$

Let $y=c t^{d}$, we have $t=\left(\frac{y}{c}\right)^{\frac{1}{d}}$, hence $d t=\frac{1}{c^{\frac{1}{d}} b} y^{\frac{1}{d}-1} d y,(6)$ can be transformed into

$$
E[T(n)]=\frac{\Gamma\left(n+\frac{1}{d}\right)}{c^{\frac{1}{d}} \Gamma(n)}
$$

Where $\Gamma(n)=\int_{0}^{\infty} x^{n-1} e^{-x} d x=(n-1)$ !。

That is to say the expectation of time on test when $n$ failures happen in the NHPP is

$$
E[T(n)]=\frac{\Gamma\left(n+\frac{1}{d}\right)}{c^{\frac{1}{d}} \Gamma(n)}
$$

As above mentioned that development process of system consists of several stages with respective tests, and modification of design is introduced once failure is observed at each stage, so the reliability growth model should reflect the growth character of step-wise jump.

From (8), the relationship between the cumulative time on test $T\left(n_{i}\right)$ and the cumulative number of failures $n_{i}$ until the ith stage can be expressed as 


$$
E\left[T\left(n_{i}\right)\right]=\frac{\Gamma\left(n_{i}+\frac{1}{d}\right)}{c^{\frac{1}{d}} \Gamma\left(n_{i}\right)}
$$

According to the assumption (iii), the cumulative number of observed failure $n_{i}$ till the ith stage is

$$
n_{i}=i
$$

From (9) and (10), the expectation of time on test till the ith stage is

$$
E[T(i)]=\frac{\Gamma\left(i+\frac{1}{d}\right)}{c^{\frac{1}{d}} \Gamma(i)}
$$

Let $a=c^{\frac{1}{d}}, b=\frac{1}{d}$, then (11) can be rewritten as

Similarly, we have

$$
E[T(i)]=\frac{\Gamma(i+b)}{a \Gamma(i)}
$$

$$
E[T(i-1)]=\frac{\Gamma(i-1+b)}{a \Gamma(i-1)}
$$

By referring to (12) and (13), the expectation of time on test at the ith stage can be obtained by

$$
\tau_{i}=E[T(i)]-E[T(i-1)]=a^{-1}\left[\frac{\Gamma(i+b)}{\Gamma(i)}-\frac{\Gamma(i-1+b)}{\Gamma(i-1)}\right]
$$

From (14), the reliability growth model under the condition of In-Time corrective strategy is formulated as

$$
\lambda_{i}=\frac{a}{\frac{\Gamma(i+b)}{\Gamma(i)}-\frac{\Gamma(i-1+b)}{\Gamma(i-1)}}
$$

Where $\lambda_{i}$ denotes system failure rate at the ith stage. $b>1$ is required to guarantee the reliability growth, and $a>0$.

The point estimation $\hat{a}$ and $\hat{b}$ are obtained by maximum likelihood (ML) procedures, the ML function is

$$
L(a, b)=\prod_{i=1}^{m} \lambda_{i} e^{-\lambda_{i} \tau_{i}}
$$

Where $m$ denotes the number of test stages, $\tau_{i}$ denotes the cumulative time on test at the ith stage, and $\lambda_{i}(i=1,2, \cdots, m)$ is given in model (15).

\section{BAYESIAN HYPOTHESIS TESTING METHOD}

The objective of this paper is to construct a Bayesian hypothesis testing method to judge whether system reliability index has achieved the predefined requirement by applying reliability growth model introduced in Section 1 to incorporate test data from diverse development stages. The prior distribution is the premise to perform Bayesian statistical analysis. 


\subsection{The prior distribution of $\lambda_{i}$}

Let $\left(1, \tau_{1}\right),\left(1, \tau_{2}\right), \cdots,\left(1, \tau_{i-1}\right)$ denote test data from $i-1$ test stages respectively, according to reliability growth model (15), we can obtained point estimation $\hat{a}, \hat{b}$ by calculating (16). Then the prediction estimation of failure rate $\lambda_{i}$ is

$$
\hat{\lambda}_{i, 0}=\frac{\hat{a}}{\frac{\Gamma(i+\hat{b})}{\Gamma(i)}-\frac{\Gamma(i-1+\hat{b})}{\Gamma(i-1)}}
$$

Let $\hat{\lambda}_{i, 0}$ be the prior mean of the failure rate $\lambda_{i}$ at ith stage, $i$. e.

$$
E\left(\lambda_{i}\right)=\hat{\lambda}_{i, 0}
$$

According to the maximum entropy method, the prior distribution $\pi\left(\lambda_{i}\right)$ can de expressed as

$$
\pi\left(\lambda_{i}\right)=\frac{e^{\mu \lambda_{i}}}{\int_{0}^{+\infty} e^{\mu \lambda_{i}} d \lambda_{i}}
$$

Where $\mu$ is a parameter to be determined.

By referring to (18) and (19), the value of $\mu$ can be acquired by solving the following equation

$$
E\left(\lambda_{i}\right)=\frac{\int_{0}^{+\infty} \lambda_{i} e^{\mu \lambda_{i}} d \lambda_{i}}{\int_{0}^{+\infty} e^{\mu \lambda_{i}} d \lambda_{i}}=\hat{\lambda}_{i, 0}
$$

After the unique parameter $\mu$ is determined, we can obtain the prior distribution $\pi\left(\lambda_{i}\right)$ of $\lambda_{i}$. Hence the prior second moment of $\lambda_{i}$ can be obtained by

$$
E\left(\lambda_{i}^{2}\right)=\frac{\int_{0}^{+\infty} \lambda_{i}^{2} e^{\mu \lambda_{i}} d \lambda_{i}}{\int_{0}^{+\infty} e^{\mu \lambda_{i}} d \lambda_{i}}
$$

As conjugate prior distribution has the advantages of tractability in mathematical computation and practical meanings for its parameters, we use Gamma distribution $G a\left(a_{i, 0}, b_{i, 0}\right)$ as the approximate distribution to fit the prior distribution $\pi\left(\lambda_{i}\right)$. Thus both $G a\left(a_{i, 0}, b_{i, 0}\right)$ and $\pi\left(\lambda_{i}\right)$ must have the same prior first and second moments. Hence

$$
\left\{\begin{array}{c}
\frac{a_{i, 0}}{b_{i, 0}}=E\left(\lambda_{i}\right) \\
\frac{a_{i, 0}\left(a_{i, 0}+1\right)}{b_{i, 0}^{2}}=E\left(\lambda_{i}^{2}\right)
\end{array}\right.
$$

By solving above equations, we get 


$$
\left\{\begin{array}{l}
a_{i, 0}=\frac{\left[E\left(\lambda_{i}\right)\right]^{2}}{E\left(\lambda_{i}^{2}\right)-\left[E\left(\lambda_{i}\right)\right]^{2}} \\
b_{i, 0}=\frac{E\left(\lambda_{i}\right)}{E\left(\lambda_{i}^{2}\right)-\left[E\left(\lambda_{i}\right)\right]^{2}}
\end{array}\right.
$$

Thus, the prior distribution $G a\left(a_{i, 0}, b_{i, 0}\right)$ of $\lambda_{i}$ at ith stage is obtained by applying the reliability growth model (15) to incorporate test data from $i-1$ development stages, where $a_{i, 0}, b_{i, 0}$ are given by (23).

\subsection{Bayesian hypothesis testing method}

Let $\theta$ denotes system MTBF. $\theta_{U}$ is the upper limit (if $\theta$ surrounds upper limit closely, products will be accepted by high probability), $\theta_{L}$ is the lower limit (if $\theta$ surrounds lower limit closely, products will be rejected by high probability), $\theta_{U}$ and $\theta_{L}$ are given in development contract, and $\theta_{U}>\theta_{L} . \alpha_{\pi_{0}}$ denotes producer risk, $\beta_{\pi_{1}}$ denotes consumer risk. The hypothesis testing problem need to be researched is to judge that $\theta_{i}$ satisfies $\theta_{U}$ or $\theta_{L}$ when development process has come to the ith stage and takes In-Time corrective strategy into account, by utilizing test data from $i$ stages under the condition of given $\alpha_{\pi_{0}}, \beta_{\pi_{1}}, \theta_{U}, \theta_{L}$. So the hypotheses can be formulated as

$$
H_{0}: \theta_{i}=\theta_{U}, \quad H_{1}: \theta_{i}=\theta_{L}
$$

Where $\theta_{i}$ denotes system MTBF at the ith stage.

To convenient calculation, the hypothesis testing about MTBF can be transformed into hypothesis testing about failure rate. So (24) is equivalent to

$$
H_{0}: \lambda_{i}=\lambda_{L}, \quad H_{1}: \lambda_{i}=\lambda_{U}
$$

Where $\lambda_{i}$ denotes system failure rate at the ith stage, $\lambda_{L}=1 / \theta_{U}, \lambda_{U}=1 / \theta_{L}, \lambda_{L}<\lambda_{U}$.

According to the Bayesian principle, the prior probability of null hypothesis $H_{0}$ from (25) is

$$
P\left(H_{0}\right) \triangleq \pi_{0}=P\left\{\lambda_{L} \mid\left(a_{i, 0}, b_{i, 0}\right)\right\}=\frac{P\left(\lambda_{L}\right) P\left\{\left(a_{i, 0}, b_{i, 0}\right) \mid \lambda_{L}\right\}}{P\left(\lambda_{L}\right) P\left\{\left(a_{i, 0}, b_{i, 0}\right) \mid \lambda_{L}\right\}+P\left(\lambda_{U}\right) P\left\{\left(a_{i, 0}, b_{i, 0}\right) \mid \lambda_{U}\right\}}
$$

Where $P\left(\lambda_{L}\right), P\left(\lambda_{U}\right)$ donote prior probability of $\lambda_{L}, \lambda_{U}$ respectively.

Here let $P\left(\lambda_{L}\right)=P\left(\lambda_{U}\right)=0.5$, thus the above prior probability of $H_{0}$ is 


$$
\begin{aligned}
\pi_{0}= & \frac{P\left\{\left(a_{i, 0}, b_{i, 0}\right) \mid \lambda_{L}\right\}}{P\left\{\left(a_{i, 0}, b_{i, 0}\right) \mid \lambda_{L}\right\}+P\left\{\left(a_{i, 0}, b_{i, 0}\right) \mid \lambda_{U}\right\}} \\
= & \frac{\frac{1}{a_{i, 0} !}\left(\lambda_{L} a_{, 0}\right)^{a_{i, 0}} \mathrm{e}^{-\lambda_{L} b_{i, 0}}}{\frac{1}{a_{i, 0} !}\left(\lambda_{L} b_{i, 0}\right)^{a_{i, 0}} \mathrm{e}^{-\lambda_{L} b_{i, 0}}+\frac{1}{a_{i, 0} !}\left(\lambda_{U} b_{i, 0}\right)^{a_{i, 0}} \mathrm{e}^{-\lambda_{U} b_{i, 0}}} \\
= & \frac{1}{1+\left(\frac{\lambda_{U}}{\lambda_{L}}\right)^{a_{i, 0}} \mathrm{e}^{\left(\lambda_{L}-\lambda_{U}\right) b_{i, 0}}}
\end{aligned}
$$

So the prior probability of alternative hypothesis $H_{1}$ is

$$
P\left(H_{1}\right) \triangleq \pi_{1}=1-\pi_{0}
$$

From the assumption (iii), the likelihood function of test data from $i$ stages is

$$
L\left\{\left(1, \tau_{i}\right) \mid \lambda_{i}\right\}=\lambda_{i} e^{-\lambda_{i} \tau_{i}}
$$

So the posterior probability ratio of hypothesis testing (25) is

$$
\begin{aligned}
O_{i} & =\frac{P\left\{H_{1} \mid\left(1_{i}, \tau_{i}\right)\right\}}{P\left\{H_{0} \mid\left(1_{i}, \tau_{i}\right)\right\}}=\frac{P\left(H_{1}\right) L\left\{\left(1, \tau_{i}\right) \mid \lambda_{U}\right\}}{P\left(H_{0}\right) L\left\{\left(1, \tau_{i}\right) \mid \lambda_{L}\right\}} \\
& =\frac{\pi_{1}}{\pi_{0}} \frac{\lambda_{U} e^{-\lambda_{U} \tau_{i}}}{\lambda_{L} e^{-\lambda_{L} \tau_{i}}}=\frac{\pi_{1}}{\pi_{0}} \frac{\lambda_{U}}{\lambda_{L}} \exp \left[\tau_{i}\left(\lambda_{L}-\lambda_{U}\right)\right]
\end{aligned}
$$

To perform the hypothesis testing, a decision threshold $A_{i}$ is introduced here, and the decision criteria are as follows:

(i) If $O_{i} \leq A_{i}$ accept null hypothesis $H_{0}$;

(ii) If $O_{i}>A_{i}$, accept alternative hypothesis $H_{1}$.

The successive question we have to solve is to calculate $A_{i}$. It is appropriate to consider computation of the two types of risks for statistical testing according to the above decision criteria before determining $A_{i}$. The type I risk, namely producer risk $\alpha_{\pi_{0}}$ is

$$
\alpha_{\pi_{0}}=P\left(O_{i}>A_{i} \mid \lambda_{i}=\lambda_{L}\right) P\left(H_{0}\right)
$$

As for $P\left(O_{i}>A_{i} \mid \lambda_{i}=\lambda_{L}\right)$, we have

$$
\begin{aligned}
& P\left(O_{i}>A_{i} \mid \lambda_{i}=\lambda_{L}\right) \\
& =P\left\{\frac{\pi_{1}}{\pi_{0}} \frac{\lambda_{U}}{\lambda_{L}} \exp \left[\tau_{i}\left(\lambda_{L}-\lambda_{U}\right)\right]>A_{i} \mid \lambda_{i}=\lambda_{L}\right\} \\
& =P\left\{\exp \left[\tau_{i}\left(\lambda_{L}-\lambda_{U}\right)\right]>A_{i} \frac{\pi_{0}}{\pi_{1}} \frac{\lambda_{L}}{\lambda_{U}} \mid \lambda_{i}=\lambda_{L}\right\} \\
& \quad=P\left\{\tau_{i}\left(\lambda_{L}-\lambda_{U}\right)>\ln A_{i}+\ln \frac{\pi_{0}}{\pi_{1}}+\ln \frac{\lambda_{L}}{\lambda_{U}} \mid \lambda_{i}=\lambda_{L}\right\}
\end{aligned}
$$

Because $\lambda_{L}<\lambda_{U}$, so (32) can be transformed into 


$$
\begin{aligned}
& P\left(O_{i}>A_{i} \mid \lambda_{i}=\lambda_{L}\right) \\
& \quad=P\left\{\tau_{i}<\frac{1}{\lambda_{L}-\lambda_{U}}\left(\ln A_{i}+\ln \frac{\pi_{0}}{\pi_{1}}+\ln \frac{\lambda_{L}}{\lambda_{U}}\right) \mid \lambda_{i}=\lambda_{L}\right\}
\end{aligned}
$$

According to the assumption (iv), we know time on test $\tau_{i}$ conforms to Gamma distribution $G a\left(1_{i}, \lambda_{i}\right)$, so we have

$$
P\left(O_{i}>A_{i} \mid \lambda_{i}=\lambda_{L}\right)=\lambda_{L} \int_{0}^{\frac{1}{\lambda_{L}-\lambda_{U}}\left(\ln A_{i}+\ln \frac{\pi_{0}}{\pi_{1}}+\ln \frac{\lambda_{L}}{\lambda_{U}}\right)} e^{-\lambda_{L} x} d x
$$

From (31) and (34), the producer risk can be obtained as

$$
\alpha_{\pi_{0}}=\pi_{0} \lambda_{L} \int_{0}^{\frac{1}{\lambda_{L}-\lambda_{U}}\left(\ln A_{i}+\ln \frac{\pi_{0}}{\pi_{1}}+\ln \frac{\lambda_{L}}{\lambda_{U}}\right)} e^{-\lambda_{L} x} d x
$$

Similarly, type II risk, namely consumer risk is

$$
\begin{aligned}
& \beta_{\pi_{1}}=P\left(O_{i} \leq A_{i} \mid \lambda_{i}=\lambda_{U}\right) P\left(H_{1}\right) \\
& =\pi_{1}\left[1-P\left(O_{i}>A_{i} \mid \lambda_{i}=\lambda_{U}\right)\right]
\end{aligned}
$$

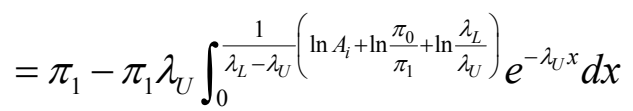

From (35) and (36), we can find that $\alpha_{\pi_{0}}, \beta_{\pi_{1}}$ are both functions on $A_{i}$, and $\alpha_{\pi_{0}}$ is the nonincreasing function on $A_{i}$, while $\beta_{\pi_{1}}$ is the non-decreasing function on $A_{i}$. Therefore, given $\alpha_{\pi_{0}}$, there exists $A_{i, 0}$ that satisfies $\alpha_{\pi_{0}}\left(A_{i, 0}\right)=\alpha_{\pi_{0}}$. At the same time, for any $A_{i}$ within the interval $\left[A_{i, 0}, \infty\right), \alpha_{\pi_{0}}\left(A_{i}\right) \leq \alpha_{\pi_{0}}$ holds, where $\alpha_{\pi_{0}}\left(A_{i}\right)$ denotes the probability of type I risk when the decision threshold is $A_{i}$. Similarly, there is $A_{i}$ which satisfies $\beta_{\pi_{1}}\left(A_{i, 1}\right)=\beta_{\pi_{1}}$ when $\beta_{\pi_{1}}$ is given, and for any $A_{i}$ within the interval $\left(0, A_{i, 1}\right], \beta_{\pi_{1}}\left(A_{i}\right) \leq \beta_{\pi_{1}}$ holds. Where $\beta_{\pi_{1}}\left(A_{i}\right)$ denotes the probability of type II risk when the decision threshold is $A_{i}$. Given the probabilities of the two types of risks, the decision threshold $A_{i}$ will be determined by the following procedure:

(i) if $A_{i, 0} \leq A_{i, 1}$, any $A_{i} \in\left[A_{i, 0}, A_{i, 1}\right]$ can be chosen as the decision threshold. The real probabilities of the two types of risks are $\alpha_{\pi_{0}}^{\prime}\left(A_{i}\right), \beta_{\pi_{1}}^{\prime}\left(A_{i}\right)$ respectively.

(ii) if $A_{i, 0}>A_{i, 1}, A_{i}$ can be determined by consulting the producer and consumer. When we choose $A_{i}$ that meets the requirement of (35), it should also minimize the value of (36), so the decision threshold can be chosen as $A_{i}=A_{i, 0}$. In the same manner, when we choose $A_{i}$ which meets the requirement of (36), it should also minimize the value of (35), so the decision threshold can be chosen as $A_{i}=A_{i, 1}$.

To sum up, the Bayesian hypothesis testing procedure for system failure rate $\lambda_{i}$ at the ith stage when system development process takes In-Time corrective strategy into account is shown as the following steps:

Step1 Obtain prior distribution of $\lambda_{i}$ by applying reliability growth model (15) to incorporate test data from previous $i-1$ test stages.

Step2 Calculate the posterior probability ratio $O_{i}$ and decision threshold $A_{i}$. 
Step3 Make a decision according to the criteria:

(i) If $O_{i} \leq A_{i}$, terminate the development process and accept null hypothesis $H_{0}$.

(ii) If $O_{i}>A_{i}$, accept alternative hypothesis $H_{1}$, improve system design and release improved system into new test stage until system failure rate has reached upper limit.

\section{NUMERICAL EXAMPLE}

A system experiences a series of test stages during development and its lifetime follows exponential distribution. The upper limit of system MTBF is 40 hours, the lower limit of system MTBF is 32 hours, and the producer risk and consumer risk are both set to be 0.2 . The problem is to judge which requirement the system MTBF has reached.

The test data is in Table 1 which lists the simulated test data derived by sampling from the NHPP when the truth values of the MTBF are known.

Table 1 test data

\begin{tabular}{llllll}
\hline stage & 1 & 2 & 3 & 4 & 5 \\
\hline $\begin{array}{l}\text { Truth value of } \\
\text { MTBF }\end{array}$ & 8.9551 & 16.2524 & 22.8743 & 29.0875 & 35.0133 \\
Time on test(h) & 8.3278 & 15.7918 & 21.4445 & 34.8337 & 35.9414 \\
\hline
\end{tabular}

To simplify the computation, the question of above hypothesis testing for the MTBF can be transformed into the testing for the failure rate, namely, we will accept system by high probability when failure rate approaches to $\lambda_{L}=0.025$; otherwise we will reject system by high probability when failure rate approaches to $\lambda_{U}=0.0313$. According to test data from previous 4 stages, by referring to (16), we can obtain the ML estimate of reliability growth model parameters $\hat{a}=0.1892, \hat{b}=1.8125$, and the prediction estimate of the failure rate $\lambda_{5}$ is $\hat{\lambda}_{5,0}=0.0287$ through (17). So the prior probability of null hypothesis is $\pi_{0}=0.4456$ by referring to (18)-(27), and the posterior probability ratio is $O_{5}=1.2420$ by referring to (30). The corresponding decision threshold are $A_{5,0}=1.1461, A_{5,1}=1.0765$ respectively when two types of risks are both 0.2. As $A_{5,0}>A_{5,1}$, we choose the decision threshold as $A_{5}=1.1461$ after consulting between consumer and producer, and the truth probabilities of the two types of risks are $\alpha_{\pi_{0}}^{\prime}=0.2, \beta_{\pi_{1}}^{\prime}=0.2814$,respectively. Since $O_{5}>A_{5}$, we reject system and demand design modification before starting the next test stage. After improving the system design and performing the sixth stage test, the observed test data is shown in Table2. Now we continue to determine which requirment the improved system MTBF has achieved.

Table2 test data at the $6^{\text {th }}$ stage

\begin{tabular}{llll}
\hline stage & $\begin{array}{l}\text { Truth value of } \\
\text { MTBF }\end{array}$ & Time on test(h) & $\begin{array}{l}\text { The number of fail- } \\
\text { ure }\end{array}$ \\
\hline 6 & 40.7196 & 42.0064 & 1 \\
\hline
\end{tabular}

Same with above calculation procedure, the ML estimate of reliability growth model parameters are $\hat{a}=0.2311, \hat{b}=1.8868$ according to test data from previous 5 stages, and the prediction estimate of the failure rate $\lambda_{6}$ is $\hat{\lambda}_{6,0}=0.0252$. So the prior probability of null hypothesis is $\pi_{0}=0.5063$, and the posterior probability ratio is $O_{6}=0.9370$. The corresponding decision threshold are $A_{6,0}=1.1549, A_{6,1}=0.9468$ respectively when the probabilities of the 
two types of risks are both 0.2 . As $A_{6,0}>A_{6,1}$, we choose the decision threshold as $A_{6}=1.1549$, and the truth probabilities of the two types of risks are $\alpha_{\pi_{0}}^{\prime}=0.2, \beta_{\pi_{1}}^{\prime}=0.2814$, respectively. Since $O_{6}<A_{6}$, the system can be accepted and the development process can be terminated. Actually, the truth value of MTBF (40. 7196) at the 6th test stage indeed more approached to upper limit, so the decision results concluded from our methods coincide with the real condition.

\section{CONCLUSION}

- A reliability growth model to describe reliability growth trend of system under the condition of In-Time corrective strategy is proposed, which well describes the features of stepwise trend of system reliability growth.

- A reliability test demonstration method is presented, which especially adapts to expensive system with destructive test when development process takes In-Time corrective strategy to modify failure. The method makes use of test data from development stages, offsets the absence of information, and effectively enhances the credibility of statistical decision.

\section{REFERENCES}

[1] A. Fries. Discrete reliability-growth models based on learning-curve property. IEEE Trans. on Reliability, 1993, 42(21): 303-306.

[2] A. Fries, A. Sen. A survey of discrete reliability-growth models. IEEE Trans. on Reliability, 1996, 45(4): 582-604.

[3] Y. Y. Xing, X. Y. Wu, P. Jiang, Q. Liu. Dynamic Bayesian evaluation method for system reliability growth based on In-Time correction. IEEE Trans. on Reliability, 2010, 59(2): 309-312.

[4] J. B. HaII, A. Mosleh. An analytical framework for reliability growth of one-shot systems. Reliability Engineering and System Safety, 2008, 93: 1751-1760.

[5] G. Pulcini. An exponential reliability-growth model in multicopy testing program. IEEE Trans. on Reliability, 2001, 50(4): 365-373.

[6] M. Giorgio, M. Guida, G. Pulcini. Reliability-growth analysis of locomotive electrical equipment. Journal of quality technology, 2006, 38(1):14-29.

[7] G. Pulcini. Correction to "how to model reliability-growth when times of design modifications are known". IEEE Trans. on Reliability, 2002, 51(2): 252-253.

[8] R. Calabfia, M. Guida, G. Pulcini. A Bayes procedure for estimation of current system reliability. IEEE Trans. on Reliability, 1992, 41(4): 616-620.

[9] R. Calabria, M. Guida, G. Pulcini. A reliability-growth mode in a Bayes-decision framework. IEEE Trans. on Reliability, 1996, 45(3): 505-510.

[10] Z. Q. Yan, X. X. Li, H. W. Xie, et al. Bayesian synthetic evaluation of multistage reliability growth with instant and delay fix modes. Journal of systems engineering and electronics, 2008, 19(6):1287-1294. 
[11] Y. Y. Xing, X. Y. Wu. Bayesian sequential testing for exponential life system with reliability growth. Journal of systems engineering and electronics, 2011, 22(6):10231029 .

This work was supported by the National Science Foundation of China (71401170, 71371182) and the Research Fund for the Doctoral Program of Higher Education of China (20134307120026). 